\title{
Expresión de Conceptos Químicos Mediante Lenguaje Significativo
}

\author{
I. Galache y P. Pérez \\ Universidad de Málaga, Facultad de Ciencias de la Educación, Área de Química Inorgánica, \\ Campus Universitario de Teatinos, 29071 Málaga-España (e-mail: igalache@uma.es)
}

\begin{abstract}
Resumen
El objetivo de este trabajo es fomentar el uso del lenguaje significativo para definir conceptos químicos en estudiantes universitarios. Se ensaya una metodología activa y participativa, que consiste en sondeo inicial, exposición en clase de los aspectos fundamentales, trabajo personal, debate y aclaración de conceptos. Se exploran dos temas: establecer la diferencia entre elementos y compuestos y el distinto comportamiento de elementos metálicos y no metálicos. Se constata en la exposición de los resultados experimentales y en la elaboración de de unidades didácticas una mayor precisión en el uso del lenguaje químico y una razonada exposición del comportamiento de las sustancias. Se concluye que la metodología es válida, ya que los alumnos se implican activamente en el proceso de aprendizaje y valoran positivamente la experiencia.
\end{abstract}

Palabras clave: enseñanza de la química, lenguaje significativo, conceptos químicos, técnicas de enseñanza

\section{Expression of Chemical Concepts by Means of Significant Language}

\begin{abstract}
The objective of this study was to promote among university students the use of significant language to define chemical concepts. An active and participative methodology which includes initial survey, class exposition of fundamental concepts, personal work, debate and clarification of concepts, was tested. Two subjects were explored: the definition of differences between elements and compounds, and the different behaviour between metallic and non metallic elements. Exposition of the experimental results and development of didactic units revealed that greater precision was established in the use of chemical languages as well as producing reasoned exposition on the behaviour of the substances. It is concluded that the methodology is valid since students became actively involved in the learning process and positively valued the experience.
\end{abstract}

Keywords: chemistry teaching, significant language, chemical concepts, teaching techniques

Nota: este artículo está tomado de "Información Tecnológica" [ISSN 0716-8756], vol. 12(2), 69-74 (2001) 


\section{INTRODUCCION}

Las experiencias de innovación educativa realizadas a lo largo de estos años han permitido detectar la utilización inapropiada por parte de los alumnos del lenguaje químico. No responde a los conocimientos que se suponen deben tener. Así cuando se pregunta lo que son mezclas y se les pide algún ejemplo, encontramos respuestas como ésta: "El agua es una mezcla de dos elementos, el hidrógeno y el oxígeno. En su estado puro se considera homogénea, por ser estable" (Pérez y Galache, 1998). Utilizan indiscriminadamente términos como: Mezcla, combinación, elemento, compuesto.

Esta y otras experiencias similares (Pérez y Galache, 1995; Blanco, 1995) han motivado este trabajo que trata de conseguir un aprendizaje significativo de la química. Esto implica que: "una definición solo se puede dar después de haber asegurado la comprensibilidad de los términos que en ella vienen utilizados" (Borsese, 1998). La cuestión del significado y el papel de la definición es un problema de gran interés e importancia: el carácter convencional de la definición la convierte en tanto más significativa cuando más se conozcan sus límites de validez, es decir, cuanto más se conozca el tema que se está tratando.

Lucas (1993), plantea la necesidad que tiene la investigación de buscar nuevas respuestas a viejas preguntas: ¿por qué enseñar ciencias? ¿Qué ciencia enseñar? ¿Cómo enseñar ciencias de una manera más efectiva? Estas cuestiones sugieren buscar correlaciones entre: el papel del profesor - ideas de los alumnos - naturaleza de la ciencia a transmitir.

En esta experiencia se intenta adaptar el lenguaje a la capacidad efectiva de recepción y de comprensión de los alumnos. Para ello se han tenido en cuenta aportaciones anteriores de Bell y Freyberg (1991); Cassels y Johnstone (1983); Llorens (1988) y Parker (1992).

Por delimitar de alguna manera el campo, se opta por el estudio de los elementos químicos, metales y no metales y se utiliza una metodología activa.

Como objetivos generales de este estudio se señalan: a) Innovar la práctica docente; b) Participación activa del alumno en su propio aprendizaje; c) Crear un puente entre el lenguaje químico y el que traen los alumnos, para facilitar el proceso de aprendizaje; d) Conocer el uso y significado de los conceptos químicos; e) Diferenciar elementos de compuestos y ser capaz de definirlos; f) Estudiar las propiedades de los metales y no metales y relacionarlas con su configuración electrónica; g) Comprobar experimentalmente algunas propiedades de metales y no metales; h) Despertar la curiosidad del alumno por la utilidad y aplicación de los materiales que le rodean.

El presente trabajo, se realizó durante el curso 1998/99, en los dos cuatrimestres y con los alumnos correspondientes. El ámbito de aplicación va dirigido a unos 600 alumnos, distribuidos de la siguiente forma:

1. Alumnos de la Diplomatura de Maestro, Facultad de Ciencias de la educación.

2. Alumnos de $1^{\circ}$ de la Licenciatura de Químicas, Facultad de Ciencias.

3. Alumnos de Química del Medio Ambiente, Facultad de Ciencias de la Educación.

\section{METODOLOGÍA}

Teniendo en cuenta los objetivos expuestos, se ensaya una metodología activa y participativa con la siguiente secuencia:

1) Inicio de la experiencia con un sondeo que permite detectar si los alumnos utilizan un lenguaje químico correcto para referirse a determinados conceptos y si conocen el significado de algunos términos.

2) Exposición en clase de los aspectos fundamentales, así como planteamiento del enfoque y metodología a seguir. 
3) Trabajo personal que ayuda a los alumnos a profundizar en el conocimiento de los diferentes elementos y su comportamiento químico. Realizan: experiencias de laboratorio; trabajos bibliográficos y de campo; elaboran unidades didácticas. Debaten y aclaran conceptos.

\section{RESULTADOS Y DISCUSION}

Se inicia la experiencia con un sondeo que permite conocer el uso de la terminología química por parte de los alumnos. Los cuestionarios son similares, aunque con algún matiz diferente, de acuerdo con el nivel que se presupone debería tener cada grupo.

\section{Sondeo inicial}

1. A los alumnos de $1^{\circ}$ de la Diplomatura de Maestro de Primaria se les presenta un cuestionario en el que se ha tenido en cuenta que, a juzgar por la experiencia de cursos anteriores, sus conocimientos de Química son más bien de un nivel bajo, de aquí que las preguntas sean más sencillas y útiles para su orientación profesional como maestros. Se pide clasificar una serie de sustancias en: I) Elementos y compuestos. II) Metales y no metales. III) Indicar tres características de los metales. IV) Señalar tres metales de uso industrial. V) Indicar tres elementos no metálicos importantes por su utilidad.

Los resultados expresados en $\%$ se presentan en la figura 1. Como se puede observar, las respuestas de los ítems I y II apenas se diferencias de las del grupo de Química del medio ambiente.

I. Un $68 \%$ clasifican elementos y compuestos.

II. Metales y no metales, los identifican un $45 \%$.

III. Apenas saben señalar características de los metales un $3 \%$.

IV. Metales de aplicación industrial, 22\%.

V. Conocen no metales importantes por su utilidad $6 \%$.

\section{$\square$ Bien $\mathbf{M a l}$ Incompleta $\mathbb{\$}$ No contesta}

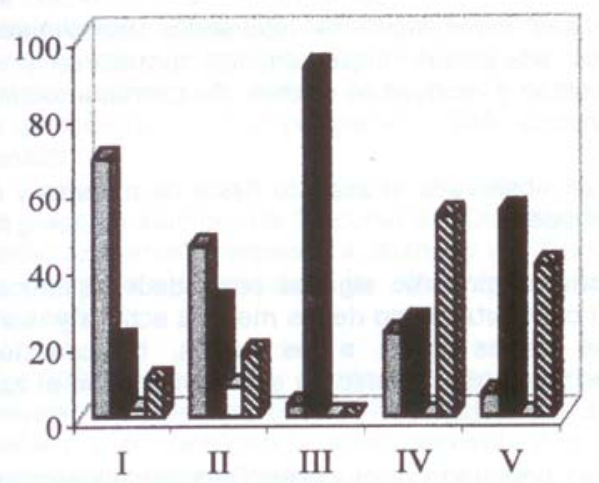

Fig. 1: Alumnos de Química de Primaria $\square$ Bien $\square$ Mal $\square$ Incompleta $\mathbf{N}$ No contesta

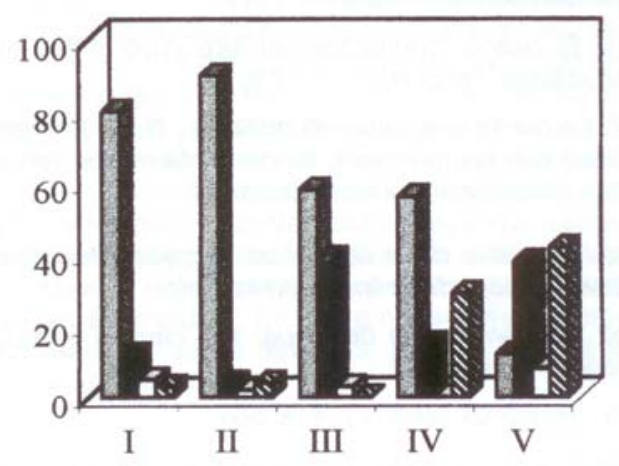

Fig. 2: Alumnos de $1^{\circ}$ de Químicas

2. En cuanto a los alumnos de $1^{\circ}$ de la Licenciatura de Químicas, en el pretest se incide más en el razonamiento de las respuestas. Es decir, no sólo se les pide que clasifiquen, sino también que definan y expliquen algunos conceptos. Se les pide clasificar una serie de sustancias en: I) Elementos y compuestos. II) Metales y no metales. III) Definir: elemento y compuesto. IV) Explicar por qué el azufre es un elemento y el metano un compuesto. V) Explicar porqué el cobre es un metal y el azufre un no metal. Los resultados se pueden observar en la figura 2. Se puede observar:

I y II. Las respuestas a los dos primeros ítems, con un 80 y $90 \%$ de aciertos, son superiores a los otros grupos. 
III. En el tercero se pide una definición de elemento y compuesto. Un 58\% responde correctamente.

IV. El cuarto "Por qué el azufre es un elemento y el metano un compuesto" responden correctamente un $58 \%$.

V. El quinto "por qué el azufre es un no metal y el cobre un metal". Respuestas correctas, un 12\%.

3. El grupos de alumnos de Química del medio ambiente 3er curso, se caracteriza por la diversidad de su currículo, ya que unos proceden de distintas especialidades de la Diplomatura de maestro y otros han optado por ella, como de libre configuración, desde diversas licenciaturas.

Están interesados en profundizar en esta materia y se ha tenido en cuenta esta motivación al realizar el pretest. Se les ha pedido clasificar una serie de sustancias en: I) Elementos y compuestos. II) Metales y no metales. III) Indicar algún elemento que consideren perjudicial para el medio ambiente. IV) Escribir tres sustancias que estén en el aire y sean importantes por su utilidad. V) Enumerar tres temas que interesen, desde el punto de vista químico, para profundizar en su conocimiento.

En la figura 3 se observa que:

I y II. Los dos primeros ítems, coinciden prácticamente con los resultados del grupo de Primaria.

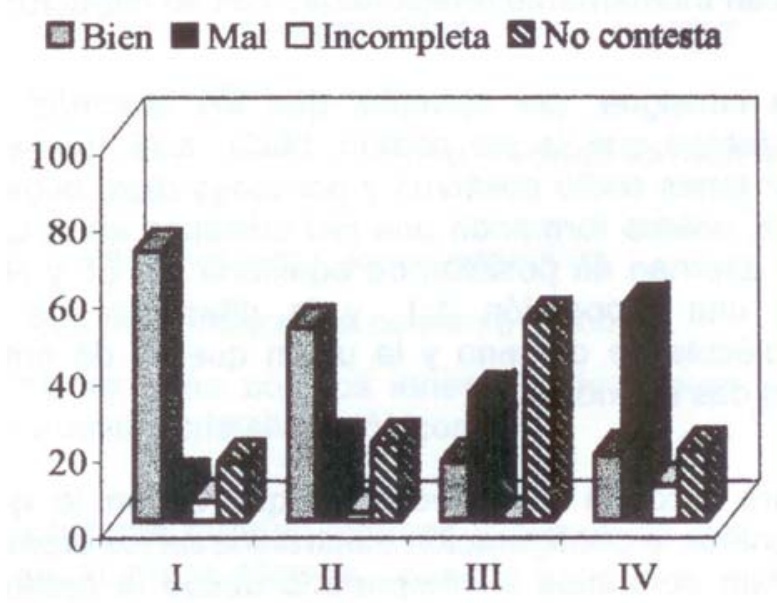

Fig. 3: Alumnos de química del medio ambiente

III. El tercero "elementos perjudiciales" lo responden correctamente un 15\%.

IV. El cuarto "elementos del aire importantes por su utilidad" responden un $17 \%$.

V. La quinta pregunta, no tabulada, puso de manifiesto que les interesan fundamentalmente temas que preocupan hoy con relación a:

a) Deterioro de la atmósfera: "agujero de ozono, lluvia ácida, "efecto invernadero", etc.

b) Contaminación del agua: sus causas y depuración de la misma.

c) Residuos sólidos y reciclados.

Temas que se han estudiado en clase y han sido objeto de trabajos personales.

Aunque entre los tres grupos no se ha pretendido establecer un paralelismo total, lo cierto es que no hay una gran diferencia en las respuestas a los dos primeros ítems que son comparables. 
Sorprende que los alumnos de la licenciatura de químicas no sean capaces de justificar por qué un elemento es metal o no metal. Tal vez una razón sea el hecho de que en esta licenciatura no hay "numerus clausus" y por tanto el nivel de química es más bajo de lo que cabría esperar.

A la vista de los resultados se ve la importancia de insistir en el trabajo personal y de equipo (experiencias de laboratorio, planteamientos de cuestiones sencillas, trabajos de campo y bibliográficos) sobre el uso correcto de la terminología ya que el lenguaje químico tiene un significado. Es decir que la afirmación de que un elemento es metal o no metal no es algo abstracto, si no que tiene un significado concreto y que la diferencia de propiedades entre ellos tiene importancia no sólo para los químicos, sino que explica el comportamiento de sustancias comunes que vemos y utilizamos cada día, precisamente porque tienen unas propiedades que están íntimamente relacionadas con su estructura.

Se consigue, por ejemplo, que los alumnos interpreten que la sal común, $\mathrm{NaCl}$, está formada por iones sodio positivos y por iones cloro negativos, unidos formando una red cristalina en la que se alternan en posición de equilibrio los $\mathrm{Cl}$ y $\mathrm{Na}$ en una proporción 1:1, y la diferencia de la molécula de oxígeno y la unión que se da entre sus dos átomos.

Para esto ha sido necesario que sepan lo que significa la configuración electrónica de los átomos y han aprendido a interpretarla desde la ordenación de los elementos en la tabla periódica. Objetivo perseguido en la realización del trabajo.

Trabajo personal y de equipo

- Las experiencias de laboratorio seleccionadas son asequibles, especialmente formativas y refuerzan y motivan el estudio. Se coordina teoría y práctica. Así:

Han observado el aspecto físico de metales y no metales.

Han comprobado algunas propiedades químicas: a) Comportamiento de los metales activos y metales nobles frente a los ácidos, b) oxidación-reducción de metales, c) experiencias con el azufre y el carbono.

Han preparado disoluciones de sales e identifican aniones y cationes por electrolisis. Aprenden así el comportamiento de los iones, qué significan y qué papel tienen el cátodo y el ánodo.

Se pide que los resultados experimentales se formulen con precisión química.

- Han realizado trabajos de campo que les permite analizar el uso adecuado del lenguaje químico:

Dos grupos de alumnos realizan análisis de libros de texto del segundo ciclo de primaria y de ESO (Enseñanza Secundaria Obligatoria), para ver cómo y cuándo introducen el tema metales y no metales. Comentan:

"Apenas se habla de algunos metales (hierro, aluminio, mercurio) y de no metales (oxígeno, carbono) pero en ningún caso se introduce o comenta la diferencia de comportamiento entre metales y no metales".

Otros dos grupos pasan un cuestionario a compañeros de distintos cursos y facultades. Señalan que:

"Un 45\% de las respuestas identifica el agua destilada y la sal común como una mezcla".

"Aproximadamente un 40\% afirma que el neón y el azufre son metales, y para un 52\% el sodio es un no metal". 
"Un 47\% afirma que es correcto decir que el agua es una mezcla de hidrógeno y oxígeno y un $70 \%$ cree que el hierro es un mineral".

Algunos utilizan indistintamente metal/mineral y comentan:

"A nosotros mismos el análisis de estos resultados nos ha hecho caer en la cuenta de los errores conceptuales y de vocabulario que tenemos, para referirnos a determinados conceptos".

Otros afirman:

"Nos ha costado pasar y clasificar los resultados del cuestionario pero hemos aprendido a diferenciar y definir, saber que el aire es una mezcla y conocer sus componentes. En definitiva, ha sido positivo realizar este trabajo para conocer el nivel de química de nuestros compañeros $y$ también el nuestro".

Un grupo de alumnos de 3er curso de "Química del medio ambiente" entrevista a alumnos del curso de orientación universitaria de diferentes institutos y señala:

"Un 50\% de alumnos confunden metales y no metales. Así clasifican el boro y el calcio como no metales y el hidrógeno como elemento fundamental componente del aire".

Por último, los que pasan el cuestionario a estudiantes de ciencias, letras y tecnológicas, establecen una comparación:

"No vemos normal que alumnos de carreras de ciencias confundan metales y no metales, quizá por que han contestado la encuesta sin poner mucha atención. Tampoco apreciamos mucha diferencia entre los alumnos de ciencias y técnicas con los de letras, tal vez porque estos conceptos, cuando se aprenden en la escuela, se olvidan, porque no se aplican o utilizan en la vida cotidiana, o porque en la escuela no se ayuda a retener con la observación y realización de experiencias no se insiste en lo que significan".

- En los trabajos bibliográficos realizados en torno a la temática de estudio, han profundizado y expuesto a sus compañeros aspectos sobre:

Descubrimiento e historia de los elementos químicos.

El sistema periódico y las propiedades periódicas de los elementos.

Propiedades generales de metales y no metales.

Estudio de algunos no metales importantes: oxígeno, nitrógeno, carbono, etc.

Metales nobles y sus propiedades.

Metalurgia de algunos metales más importantes.

El estudio de la historia permite conocer los marcos teóricos que se han utilizado para interpretar los fenómenos y puede servir de herramienta para el aprendizaje de algunos conceptos.

En el trabajo de Galache et al. (1991), se consideró que la visón histórica puede ayudar a entender las dificultades que se encuentran al pretender introducir el concepto "ión".

Es sumamente interesante acercarse a la figura de Faraday, su obra y su correspondencia (Caamaño et al., 1987). Es, ciertamente, un modelo de científico del siglo XIX. Introdujo una terminología que se sigue utilizando en la actualidad: electrólisis, electrolitos, electrodos, ánodo, cátodo, anión, catión, ión, etc. Afirmó por primera vez que los iones son partículas eléctricas cargadas que transportan electricidad. Cuidó mucho su vocabulario científico, lo que le permitió interpretar numerosos fenómenos y lo sometió a la crítica de diversos colegas revisándolo y 
contrastándolo repetidas veces. Escribe Faraday a Whewell (Pearce, 1971): "Busco algunos nombres para expresar mis experiencias en electricidad". En otro momento dice: "estoy satisfecho de estos términos, pero no con otros que he utilizado".

Esta forma de proceder de Faraday, precisa meticulosa, corrobora la importancia del lenguaje científico. Llama la atención su preocupación porque "los escolares" puedan recordar estos términos fácilmente y aparece en una de sus cartas una inquietud de carácter didáctico: que el término sea de fácil lectura, claro, preciso, que permita establecer relaciones (ánodo-anión, cátodo-catión).

Ha resultado motivador para los alumnos el interés y esfuerzo de un gran científico por utilizar una terminología adecuada. Como ha quedado de manifiesto, al elaborar unidades didácticas dirigidas a niños de $2^{\circ}$ ciclo de educación primaria, ya que han cuidado especialmente el lenguaje químico.

- Al finalizar esta experiencia, se constata que la mayoría de los alumnos llegan a interpretar:

Por qué determinados elementos del sistema periódico son metales.

Qué caracteriza a los metales.

Qué son los electrones de valencia.

Por qué los metales dan iones positivos.

Cómo se unen los átomos en los metales.

Por qué tienen brillo metálico, son buenos conductores de calor y la electricidad.

Así mismo pueden explicar:

Por qué los átomos de los no metales forman entre sí moléculas.

Por qué tienen a dar iones negativos.

Por qué no conducen la corriente eléctrica.

Cómo se unen con los iones positivos para dar compuestos cristalinos iónicos.

No obstante, quedan lagunas y sorprende que algunos alumnos afirmen "el neón es un metal porque tiene completa su capa de valencia" y al mismo tiempo indican como características de los metales "facilidad para ceder electrones".

Esta incoherencia e inconsistencia que se percibe no debe resultar extraña si se tiene en cuenta que el químico utiliza una lógica y criterios científicos, que son los que se considera "correctos" y en muchos casos, diferentes de los que utilizan los alumnos. Black y Simon (1992), se refieren a esto con el símil de "dos islas" y al papel del profesor como el de "construir puentes entre ellos".

\section{CONCLUSIONES}

A partir de los resultados obtenidos se concluye que:

1. Se ha conseguido avanzar en la comprensión de los conceptos químicos y en la adquisición del lenguaje científico adecuado.

2. Se ha llegado a diferenciar de forma razonada lo que son metales y no metales, así como sus propiedades características.

3. Se ha despertado en los alumnos el interés por los materiales que les rodean.

4. Las respuestas a las cuestiones de las pruebas escritas han sido amplias, razonadas y explícitas, así a la pregunta: 
"Por qué el azufre es un no metal y el cobre un metal", responden: "Porque el azufre tiene configuración electrónica $[\mathrm{Ne}] 3 \mathrm{~s}^{2} \mathrm{p}^{4}$, pertenece al grupo 16 , puede aceptar electrones y carece de brillo. El cobre con configuración $[\mathrm{Ar}] 3 \mathrm{~d}^{10} 4 \mathrm{~s}^{1}$, cede electrones, tiene brillo metálico y conduce la corrientes eléctrica y el calor".

5. Los alumnos se implican activamente en el proceso de aprendizaje y han superado las pruebas escritas sin dificultad. El resultado académico ha sido bueno y ellos han valorado positivamente la experiencia.

6. La metodología empleada es válida pero requiere de tiempo y dedicación.

\section{REFERENCIAS}

Bell, B. y P. Freyberg; "El lenguaje en la clase de ciencias" en El aprendizaje de las ciencias de los alumnos, de Osborne, R. y Freyberg, P. (eds.) Madrid. Narcea (1991).

Black, P. y S. Simon; "Progression in Learning Science". Research in Science Education: 22, 4554 (1992).

Blanco, A.; "Estudio de las concepciones de los alumnos sobre algunos aspectos de las disoluciones y de los factores que influyen en ellas". Tesis doctoral publicada en microficha. Universidad de Málaga (1995).

Borsese, A.; "Enseñanza, lenguaje, aprendizaje significativo: el caso de la química" en Didáctica de las Ciencias y Transversalidad. Edita Universidad Málaga (1998).

Caamaño, A., C. Maestre, C. Mayor y T. Ventura; "Historia de la química: una herramienta útil para el aprendizaje de los conceptos químicos". Enseñanza de las ciencias: extra, 161-162 (1987).

Cassels, J. y A. Johnstone; "The meaning of words and the teaching of chemistry", Education in Chemistry, January, pp.10-11 (1983).

Galache López, M.I., E. Camacho Domínguez y A. Rodríguez García; "Origen histórico del término ion". Enseñanza de las Ciencias: 9 (2), 187-192 (1991).

Llorens, J.; "Aprendizaje de la química y ejemplo del lenguaje". Enseñanza de las Ciencias: 7 (2), 195-197 (1988).

Lucas, A.; "Condicionantes del currículo y aportaciones a la práctica de la educación en ciencias" en Palacios, C; Ansoleaga, D. y Ajo, A.; (eds), Diez años de investigación e innovación en enseñanza de las ciencias. Madrid. CIDE (1993).

Parker, L.: "Language in Science education: implication for teachers". The Australian Science Teachers Journal: 38 (2), 111-119 (1992).

Pearce, L.; The selected correpondence of Michael Faraday. (ed. By L. Pearce Willians. The royal institution of great britain. University Press. Cambridge) (1971).

Pérez Miranda, P. e I. Galache López; "Una experiencia para mejorar el proceso de enseñanzaaprendizaje del concepto ácido-base" en Innovación educativa en la Universidad de Málaga. Málaga. ICE y Servicio Publicaciones Universidad (1995).

Pérez Miranda, P. e I. Galache López; "Ensayo de un método activo para el conocimiento de la materia y su comportamiento" en promover la calidad de la enseñanza Universitaria de Tojar Hurtado, J.C. y otros, Málaga. ICE y Servicio Publicaciones Universidad (1998). 